\title{
Astrometric suitability of optically-bright ICRF sources for the alignment with the future Gaia celestial reference frame ${ }^{\star}$
}

\author{
G. Bourda, P. Charlot, and J.-F. Le Campion
}

\author{
Laboratoire d'Astrophysique de Bordeaux, Université de Bordeaux, CNRS UMR5804, BP 89, 33271 Floirac Cedex, France \\ e-mail: Geraldine.Bourda@obs.u-bordeaux1.fr
}

Received 24 July 2008 / Accepted 1 August 2008

\section{ABSTRACT}

\begin{abstract}
Context. The International Celestial Reference Frame (ICRF), currently based on the position of 717 extragalactic radio sources observed by VLBI (Very Long Baseline Interferometry), is the fundamental celestial reference frame adopted by the International Astronomical Union (IAU) in 1997. Within the next 10 years, the European space astrometry mission Gaia, to be launched by 2011, will permit determination of the extragalactic reference frame directly in the visible for the first time. Aligning these two frames with the highest accuracy will therefore be very important in the future for ensuring consistency between the measured radio and optical positions.

Aims. This paper is aimed at evaluating the current astrometric suitability of the individual ICRF radio sources which are considered appropriate for the alignment with the future Gaia frame.

Methods. To this purpose, we cross-identified the ICRF and the optical catalog (Véron-Cetty \& Véron, 2006, A\&A, 455, 773), in order to identify the optically-bright ICRF sources that will be positioned with the highest accuracy with Gaia. Then we investigated the astrometric suitability of these sources by examining their VLBI brightness distribution.

Results. We identified 243 candidate ICRF sources for the alignment with the Gaia frame (i.e. with an optical counterpart brighter than the apparent magnitude 18), but only 70 of these (i.e. only $10 \%$ of the ICRF sources) are found to have the necessary high astrometric quality (i.e. a brightness distribution that is compact enough) for this link. Additionally, it was found that the QSOs (quasi stellar objects) that will have the most accurate positions in the Gaia frame tend to have less-accurate VLBI positions, most probably because of their physical structures.

Conclusions. Altogether, this indicates that identifying other high-quality VLBI radio sources suitable for the alignment with the future Gaia frame is mandatory.
\end{abstract}

Key words. astrometry - reference systems - quasars: general

\section{Introduction}

The International Celestial Reference System (ICRS) is a kinematical system that assumes that the Universe does not rotate, hence breaking the history of the inertial system materialization from observations attached to the apparent motion of the Sun (Arias et al. 1995). The International Celestial Reference Frame (ICRF) is the realization at radio wavelengths of the ICRS, through very long baseline interferometry (VLBI) measurements of extragalactic radio source positions. It was adopted by the International Astronomical Union (IAU) as the fundamental celestial reference frame during the IAU XXIIIrd General Assembly in Kyoto, Japan, in August 1997. The initial realization was based on the positions measured with VLBI of 212 defining extragalactic radio sources (setting the direction of the ICRF axes). In addition, positions for 396 other sources (divided into 294 candidate and 102 other sources) were included to make the frame denser (Ma et al. 1998). The ICRF was extended at later stages with the positions of another 109 sources commonly referred to as new sources (Fey et al. 2004). Overall, the ICRF currently consists of a set of VLBI coordinates for 717 extragalactic radio sources, most of which show submilliarcsecond accuracy. The accuracy of the individual source positions depends on the number of observations but also on the compactness and positional stability of the sources that show

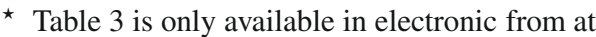
http: //www . aanda.org brightness distributions that are often not point-like and timevariable (Ma et al. 1998; Fey \& Charlot 2000).

The European space astrometry mission Gaia, to be launched by 2011, will survey about one billion stars in our Galaxy and throughout the Local Group along with 500000 quasi stellar objects (QSOs), down to an apparent optical magnitude of 20 (Perryman 2001). Optical positions with Gaia will be determined with an unprecedented accuracy, ranging from a few tens of microarcseconds ( $\mu$ as) at magnitude 15-18 (targeted accuracies are $16 \mu$ as at $15 \mathrm{mag}$ and $70 \mu$ as at $18 \mathrm{mag}$ ) to about $200 \mu$ as at magnitude 20 (Lindegren 2008). Unlike Hipparcos, Gaia will permit the construction of an extragalactic frame directly at optical wavelengths, based on the QSOs with the most accurate positions (i.e. the QSOs with magnitude brighter than 18; Mignard 2003). Mignard (2002) demonstrates that the residual spin of the Gaia frame can be determined to $0.5 \mu \mathrm{as} / \mathrm{yr}$ with a "clean sample" of about 10000 such QSOs. A preliminary Gaia catalog is expected to be available by 2015 with the final version released by 2020 .

In the future, aligning the ICRF with the Gaia celestial reference frame will be crucial for ensuring consistency between the measured radio and optical positions. This alignment will be important not only for guaranteeing the proper transition in the case that the fundamental reference frame is moved from the radio domain to the optical domain (as currently anticipated) but also for registering the radio and optical images of any celestial target with the highest accuracy. To align the two frames with the 
Table 1. Number of ICRF extragalactic radio sources cross-identified with the optical catalog of Veron-Cetty \& Véron (2006, noted VV2006), depending on the source type in this catalog and on the upper limit used for the position differences in the cross-identification.

\begin{tabular}{llrrrrr}
\hline \hline & & $5^{\prime \prime}$ & $4^{\prime \prime}$ & $3^{\prime \prime}$ & $2^{\prime \prime}$ & $1^{\prime \prime}$ \\
\hline ICRF & $\cap$ Quasars & 409 & 409 & 406 & 387 & 250 \\
& $\cap$ BL Lac & 66 & 65 & 65 & 64 & 45 \\
& $\cap$ Active galaxies & 66 & 66 & 64 & 61 & 36 \\
\hline ICRF $\cap$ VV2006 & 541 & 540 & 535 & 512 & 331 \\
\hline
\end{tabular}

highest accuracy, it is desirable to have several hundred common objects (the more common objects, the more accurate the alignment) with a uniform sky coverage and very accurate radio and optical positions. Obtaining such accurate positions implies that the link sources must have (i) an apparent optical magnitude $V$ brighter than 18 , for the highest Gaia astrometric accuracy (Mignard 2003), and (ii) no extended VLBI structures, for the highest VLBI astrometric accuracy (Fey \& Charlot 2000).

This paper is aimed at evaluating the suitability of the current individual ICRF extragalactic radio sources for the alignment with the future Gaia frame. First, the ICRF is cross-identified with the Véron-Cetty \& Véron (2006) optical catalog of QSOs in order to identify the ICRF sources with a proper optical counterpart for the Gaia link. The astrometric suitability of these sources is then investigated by examining their VLBI brightness distribution and their position accuracy in the ICRF. In this investigation, the structure index defined by Fey \& Charlot (2000) is used to identify the sources that have the most compact VLBI brightness distributions. This study results in a sample of 70 ICRF sources, which have appropriate compact structures on VLBI scales and are brighter than magnitude 18 . These sources are at present the best candidates for the ICRF-Gaia alignment.

\section{Optical counterparts of ICRF sources}

\subsection{Cross-identification of radio and optical positions}

Véron-Cetty \& Véron (2006) compiled several optical surveys of active galactic nuclei (AGN) from the literature in order to create a homogeneous, compact, and convenient catalog of 108080 objects (85221 quasars, 1122 BL Lac objects, and 21737 active galaxies). To identify the ICRF sources that have an optical counterpart, we have cross-identified the positions in this catalog with those in the ICRF. For this cross-identification, it was necessary to adopt an upper limit on the optical-radio position differences above which the corresponding radio and optical sources were considered as different objects.

We tested the impact of the selected upper limit by carrying out the cross-identification of the two catalogs for several values of this parameter, ranging from $1^{\prime \prime}$ to $5^{\prime \prime}$, the results of which are shown in Table 1. From this table, it is found that there are no major differences whether one sets the upper limit value to $3^{\prime \prime}, 4^{\prime \prime}$ or $5^{\prime \prime}$. On the other hand, significantly fewer sources are found to be common when an upper limit value of $1^{\prime \prime}$ or $2^{\prime \prime}$ is selected. Finally we decided to adopt the upper limit value of $3^{\prime \prime}$, equivalent to three times the accuracy of the optical positions in the Véron-Cetty \& Véron (2006) catalog, which is roughly at the 1 " level. Uncertainties in the VLBI positions make a negligible contribution to the position differences since such uncertainties are at the milliarcsecond level.

Based on this cross-identification, we determined that $75 \%$ of the ICRF sources (i.e. 535 sources out of a total of 717 ICRF
Table 2. Number of ICRF extragalactic radio sources cross-identified with the optical catalog of Véron-Cetty \& Véron (2006, noted VV2006), depending on the ICRF source category.

\begin{tabular}{lrrrr}
\hline \hline & \multicolumn{4}{c}{ ICRF catalog } \\
& $\begin{array}{r}\text { Defining } \\
(212)\end{array}$ & $\begin{array}{r}\text { Candidate } \\
(294)\end{array}$ & $\begin{array}{c}\text { Other } \\
(102)\end{array}$ & New \\
& & & & \\
\hline VV2006 $\cap$ ICRF & 132 & 160 & 70 & 44 \\
Quasars (406) & 27 & 24 & 11 & 3 \\
BL Lac (65) & 16 & 21 & 9 & 18 \\
Active galaxies (64) & $175 / 212$ & $205 / 294$ & $90 / 102$ & $65 / 109$ \\
\hline Total (535/717) & & & &
\end{tabular}

Note 1 . The upper limit used for the position differences in the crossidentification is $3^{\prime \prime}$.

sources) have an optical counterpart in Véron-Cetty \& Véron (2006). This set of sources includes 406 quasars (76\%), $65 \mathrm{BL}$ Lac objects (12\%), and 64 active galaxies (12\%). These results are comparable to those of Souchay et al. (2006) who used the Véron-Cetty \& Véron (2003) optical catalog for the crossidentification.

Table 2 presents the distribution of the 535 cross-identified ICRF sources, depending on their categorization in the ICRF as defining, candidate, other or new sources. This table shows that:

- $83 \%$ of the ICRF defining sources, $70 \%$ of the ICRF candidate sources, $88 \%$ of the ICRF other sources, and $60 \%$ of the ICRF new sources have an optical counterpart in VéronCetty \& Véron (2006);

- $33 \%$ of the ICRF sources having an optical counterpart in Véron-Cetty \& Véron (2006) are defining sources, 38\% candidate sources, $17 \%$ other sources, and $12 \%$ new sources.

Based on these statistics, there is no evidence for any dependence of the existence of optical counterparts on ICRF source categories. The sample of sources identified here forms the initial pool of ICRF sources from which to identify the best candidates for the Gaia link after consideration of optical magnitudes, source structure, and astrometric accuracy.

\subsection{Distribution of optical magnitudes}

In a second stage, we investigated the distribution of the apparent optical magnitude $V$ for the 535 ICRF sources identified in the previous step. Five of these sources, with undefined optical magnitudes in the Véron-Cetty \& Véron (2006) catalog, have not been considered. As shown in Fig. 1, the distribution of optical magnitudes peaks at $V$ between 18 and 19 with the bulk of the sources brighter than $V=20$. Overall, there are 485 sources brighter than 20 , i.e. detectable by Gaia, while only 45 sources are weaker than 20 . When restricting the magnitude limit to 18 , the sample is reduced by a factor of 2 , leaving only 243 sources. These 243 sources, representing $34 \%$ of the ICRF catalog, would be good candidates for establishing the alignment with the future Gaia celestial reference frame, provided they show limited structure on VLBI scales and have high VLBI positional accuracy.

\section{Astrometric suitability based on observed VLBI structure}

The quality of the alignment between the ICRF and the Gaia frame will depend strongly on the astrometric suitability of the 


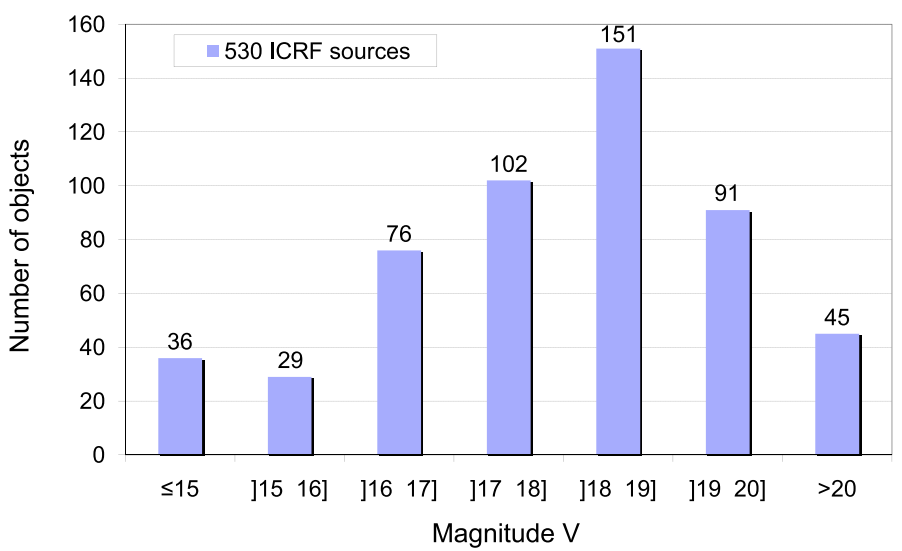

Fig. 1. Distribution of the apparent optical magnitude $V$ for the 530 ICRF extragalactic radio sources that have a Véron-Cetty \& Véron (2006) optical counterpart.

sources used for this alignment. To ensure the highest accuracy, the sources with the highest astrometric quality from the above sample should be used.

On the milliarcsecond scale, most of the extragalactic radio sources exhibit spatially extended structures that vary in both time and frequency. As shown by Charlot (1990), such structures may introduce significant errors in the VLBI measurements that deteriorate the source position accuracy. The astrometric suitability of the sources may be estimated from the observed structures through the so-called "structure index", first introduced by Fey \& Charlot (1997). The structure index ranges from 1 to 4. Structure index values of 1 or 2 point to the most compact sources with the highest astrometric quality, whereas higher values of the structure index ( 3 or 4 ) correspond to sources with extended structures, which are less suitable for astrometry. For the alignment of the ICRF and Gaia frame, only sources with structure index values of 1 or 2, i.e. with the highest astrometric quality, should be used since one wants to determine the link with the highest accuracy.

The structure index distribution for the 485 optically-bright ICRF sources with an apparent optical magnitude $V \leq 20$ (see Sect. 2) was investigated and is plotted separately for the sources with $V \leq 18$ and for those with $V$ between 18 and 20 in Fig. 2. For these plots, structure indices from the most recent calculation by Charlot et al. (2006) were used, and the distribution is shown for both $S$ and $X$ bands, the two standard frequency bands used in VLBI astrometry. Overall, structure index values are available for 410 sources at $X$ band (205 sources with $V \leq 18$ and 205 sources with $18<V \leq 20$ ) and 345 sources at $S$ band (169 sources with $V \leq 18$ and 176 sources with $18<V \leq 20$ ). Interestingly, the sources with magnitude $V \leq 18$ tend to have larger structure indices than those with $18<V \leq 20$, as noticed when comparing the corresponding distributions in Fig. 2. This indicates that the brighter optical sources have statistically more extended radio structures. Based on this analysis, it is also found that only 70 ICRF sources out of those with $V \leq 18$ have an excellent or good astrometric suitability (i.e. an $X$-band structure index value of either 1 or 2) appropriate for determining the Gaia link with the highest accuracy (see list in Table 3). This represents only a small fraction (about $10 \%$ ) of the entire ICRF catalog. The sky distribution of these 70 sources, plotted in Fig. 3, is reasonable but indicates that there is a general lack of sources.

\section{Discussion}

This paper is aimed at identifying from the current ICRF extragalactic radio sources the best candidates to establish an accurate alignment with the future Gaia frame. Figure 4 summarizes the successive steps completed to achieve this goal. We first pointed out that $75 \%$ of the ICRF sources have an optical counterpart (535 sources), while less than half of these are potential candidates for the alignment with the future Gaia frame (243 ICRF sources with $V \leq 18$, i.e. with the best positions measured by Gaia). And finally, we concluded that only $10 \%$ of the ICRF sources can be identified currently as the best candidates to establish this alignment with the highest accuracy (70 opticallybright ICRF sources with the highest astrometric quality).

To supplement this study, we also investigated the distribution of position accuracy, as available from Ma et al. (1998) and Fey et al. (2004), which is plotted in Fig. 5 for the 485 opticallybright ICRF radio sources with $V \leq 20$. From this figure, we can notice that the sources with $18<V \leq 20$ appear to have higher positional accuracy than those with $V \leq 18$. This is especially noticeable when comparing the fraction of sources with position accuracy better than 0.5 mas and those with positional accuracy in the range $0.5-1$ mas. This fraction is $71 \%$ for the sources with $18<V \leq 20$ (135 sources out of a total of 190 sources with position accuracy better than 1 mas), whereas it is $55 \%$ for the sources with $V \leq 18$ (98 sources out of a total of 177 sources with position accuracy better than 1 mas). This trend is also confirmed when comparing the median positional accuracy, which is 0.60 mas for the sources with $V \leq 18$ and 0.47 mas for the sources with $18<V \leq 20$. Such a finding is consistent with our results on astrometric suitability (Sect. 3), which indicate that the brighter sources $(V \leq 18)$ have statistically more extended VLBI structures. It is thus likely that the deteriorated position accuracy for the $V \leq 18$ sources in Fig. 5 is a result of their having larger structures. A similar correlation between source structure and position accuracy was also reported by Fey \& Charlot (2000). In the context of Gaia, this means that the sources that will have the most accurate optical positions measured by Gaia are not statistically those that have the best measured positions in the ICRF. It will thus be crucial in the future to unveil more high-quality optically-bright radio sources suitable for the Gaia link beyond those currently identified from the ICRF and reported in Table 3.

A first opportunity to find such sources may come from the second generation of the ICRF (ICRF-2), currently under development, which will improve the position accuracy of the current ICRF sources and provide VLBI positions of additional sources. This new frame will rely on data acquired by international observing programs organized through the International VLBI Service for Geodesy and Astrometry (Schluter \& Behrend 2007) or other programs such as that developed in the southern hemisphere by the United States Naval Observatory (Ojha et al. 2004, 2005). Another option would be to use the Very Long Baseline Array (VLBA) Calibrator Survey (VCS), which comprises milliarcsecond accurate positions for about 3000 additional sources along with images for a large fraction of those sources (Beasley et al. 2002; Fomalont et al. 2003; Petrov et al. 2005, 2006; Kovalev et al. 2007; Petrov et al. 2008). Finally, an additional possibility is to search for new VLBI sources, by targeting radio sources weaker than those observed so far in VLBI astrometry (i.e. with flux densities typically below $100 \mathrm{mJy}$ ). Such sensitive observations can now be realized owing to recent increases in the VLBI network sensitivity (e.g. recording at $1 \mathrm{~Gb} / \mathrm{s}$ ) and by using a network comprising large antennas like the European VLBI Network (EVN) (Charlot 2004). This 

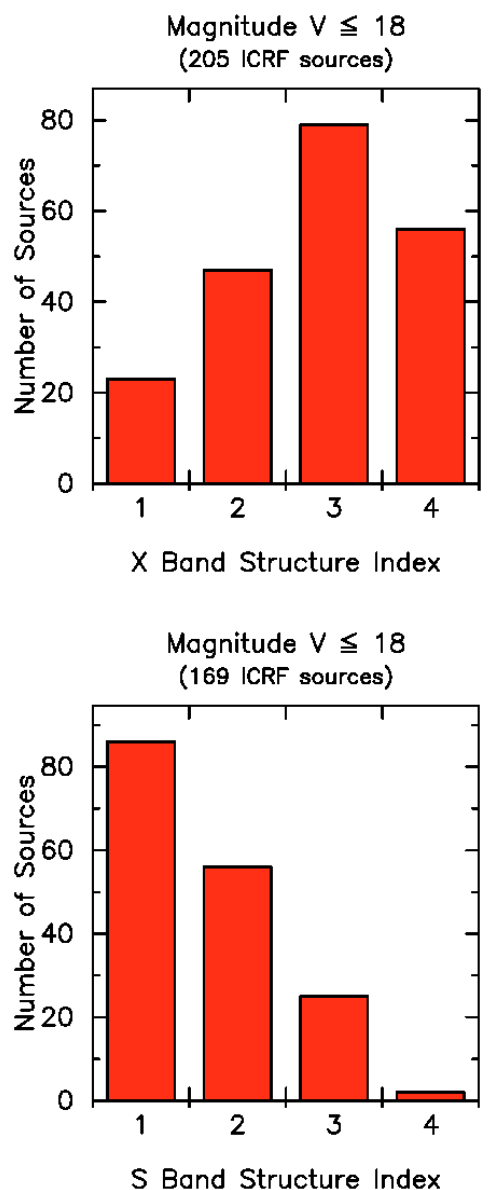

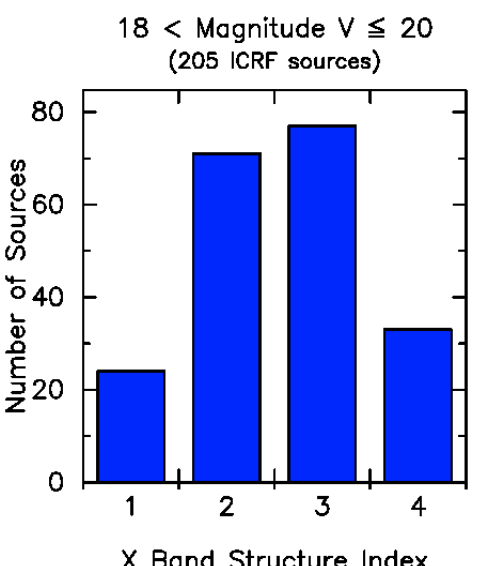

$18<$ Magnitude $V \leqq 20$ (176 ICRF sources)

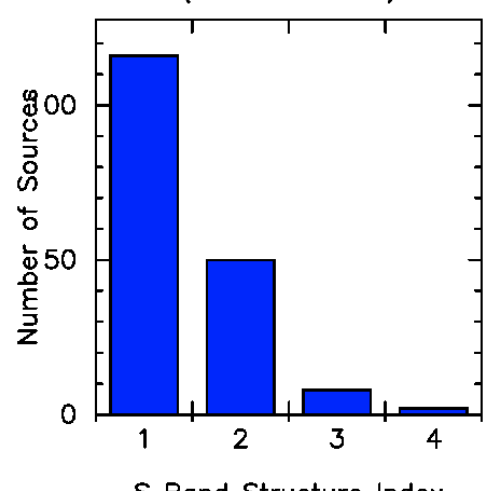

$S$ Band Structure Index

Fig. 2. Distribution of the $X$ - and $S$-band structure indices among the optically bright ICRF sources; upper panel: $X$-band structure index distribution; lower panel: $S$-band structure index distribution; left-hand side: sources with $V \leq 18$; right-hand side: sources with $18<V \leq 20$.

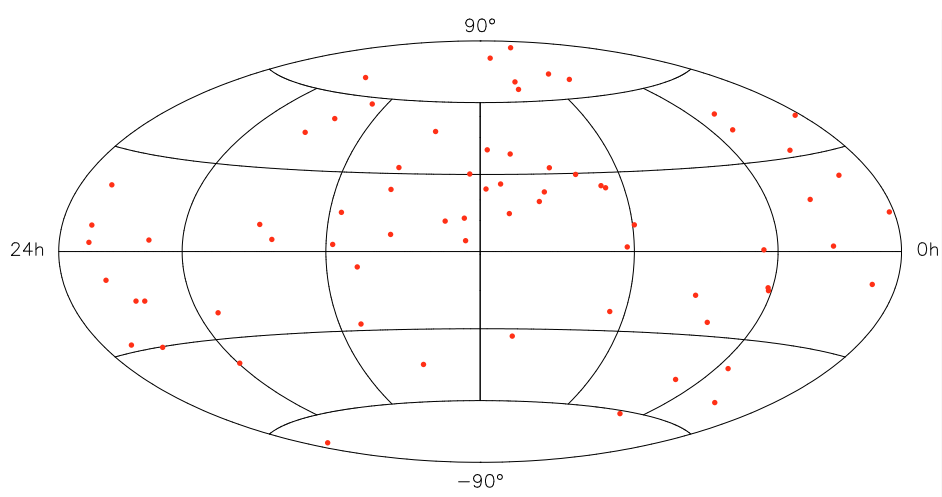

Fig. 3. Sky distribution for the 70 ICRF sources suitable for the alignment with the future Gaia frame.

identification of new optically-bright VLBI sources for the Gaia frame alignment is the purpose of an observational program initiated in 2007 which focuses on 450 weak radio sources selected from the NVSS (NRAO VLA Sky Survey; Condon et al. 1998) and brighter than the apparent optical magnitude 18. In the initial step, about $90 \%$ of these weak radio sources have been detected during an EVN pilot experiment (Bourda et al. 2008), hence drawing good prospects for increasing the pool of VLBI extragalactic radio sources suitable for aligning the ICRF and the future Gaia frame with the highest accuracy.

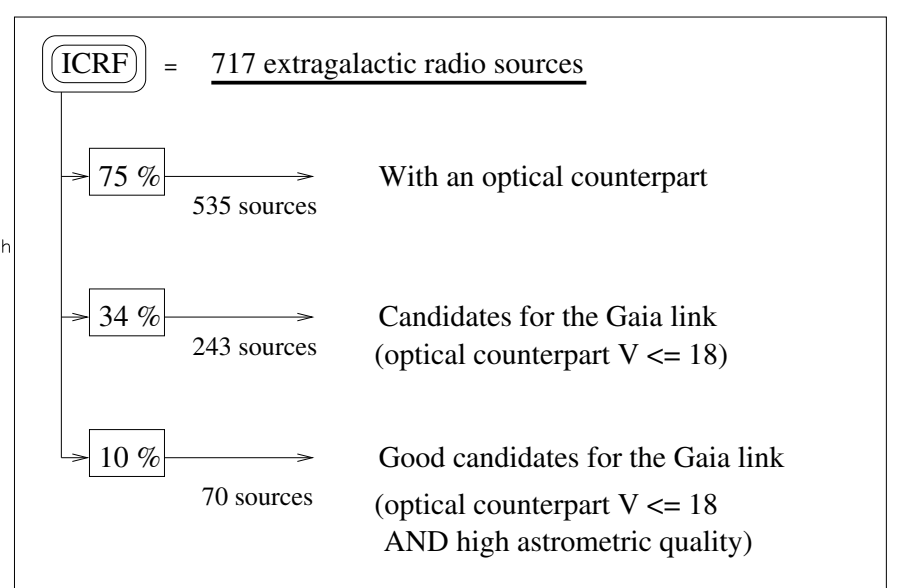

Fig. 4. Summary of the successive selection steps conducted to identify the best ICRF sources candidates for the alignment between the current ICRF and the future Gaia frame.

Another issue that might affect the choice of sources for this alignment is the possible variability of the apparent magnitude for the extragalactic sources observed at optical wavelengths. These variations are common and can easily reach one magnitude unit, especially for BL Lac objects that are known to vary on short time scales, as illustrated in Fig. 6. Such variability may affect the selection of candidate sources for the alignment of the ICRF and the Gaia frame if only a limited number of measurements are available for their optical magnitude. For this reason, it 


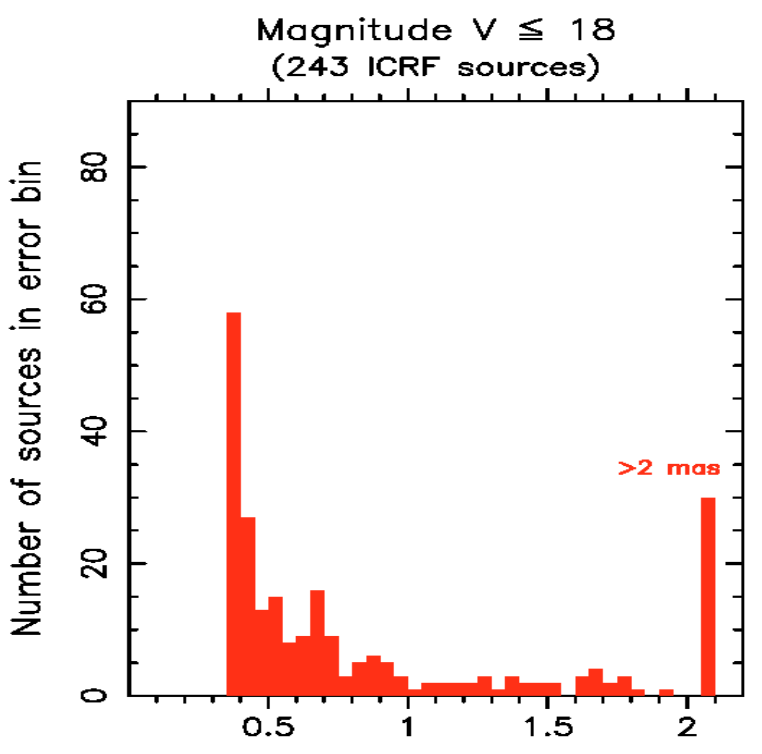

Arc length error (mas)

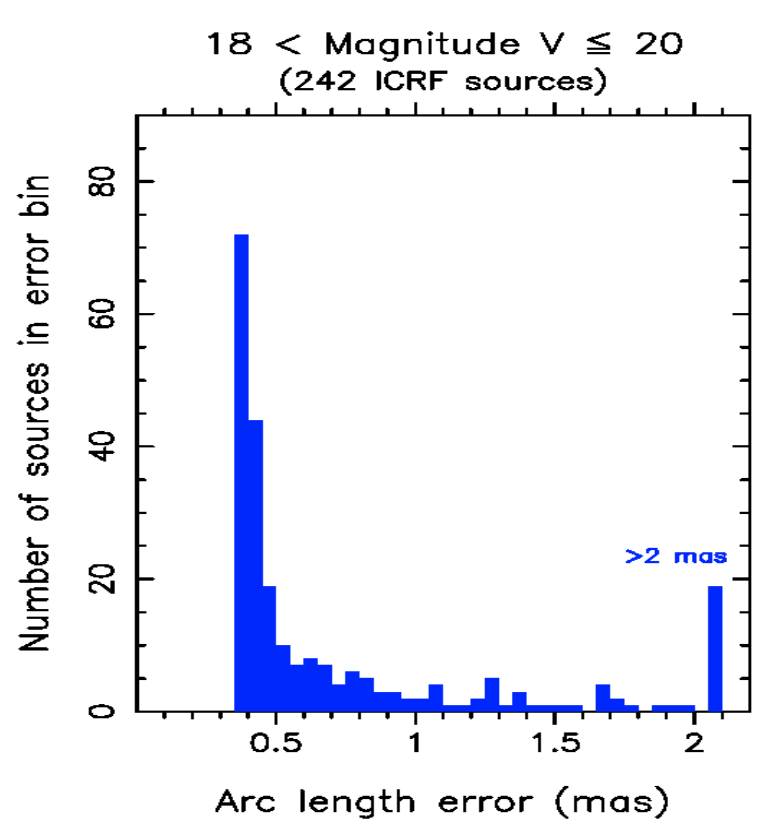

Fig. 5. Distribution of position accuracy for the 485 ICRF sources with an optical apparent magnitude $V$ brighter than 20. The accuracy is calculated as $\sqrt{\sigma_{\alpha}^{2} \cos ^{2} \delta+\sigma_{\delta}^{2}}$ where $\sigma_{\alpha}$ and $\sigma_{\delta}$ are the errors in right ascension and declination, as taken from the ICRF catalog. The upper panel shows the positional accuracy for the 243 sources with $V \leq 18$, while the lower panel shows the positional accuracy for the 242 sources with $18<V \leq 20$.

is desirable to engage in a long term optical monitoring on these candidate sources, especially those that have a magnitude near 18 , to determine if on average they have an optical magnitude that is brighter or weaker than 18 . While the results of this monitoring might impact at some level the list of sources in Table 3, this should not significantly change the percentage of suitable ICRF sources currently available for the Gaia frame alignment.

Finally, it is important to note that obtaining the alignment between the ICRF and the future Gaia frame with the highest accuracy is essential, not only to ensure the consistency between the measured radio and optical positions, but also to pinpoint the

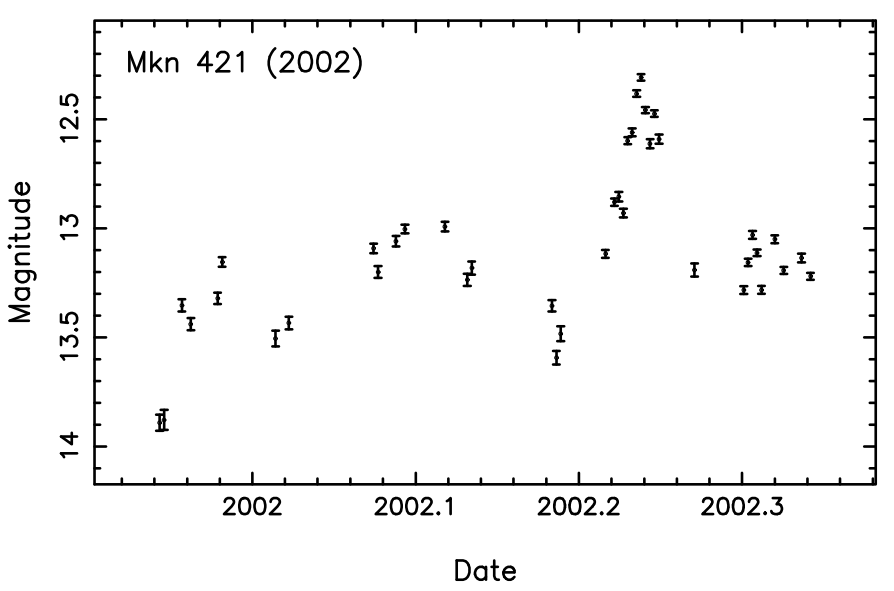

Fig. 6. Variation in the apparent optical magnitude of the BL Lac object Mkn $421(1101+384)$ from the ICRF, during the year 2002, from optical monitoring at Bordeaux Observatory (Charlot \& Le Campion 2004).

relative location of the optical and radio emission in AGNs to a few tens of $\mu$ as. In fact, the optical and radio emission may not always be spatially coincident at this level of accuracy due to opacity effects in AGN jets. For example, Kovalev et al. (2008) shows that on average the optical-radio core shift is at the level of $100 \mu$ as from studying a sample of 29 objects, which is significant considering the expected accuracy of the Gaia catalog and the one foreseen for the ICRF by $2015-2020$. It will thus be important to use a large number of objects in aligning the two frames so that positional inconsistencies are averaged out. On the other hand, the differences between the optical and radio positions may provide a direct measurement of such core shifts, which would be of high interest for probing AGN jets properties.

\section{Conclusion}

This study focused on the astrometric suitability of the current ICRF extragalactic radio sources for the alignment with the future Gaia frame. We identified 243 candidate sources for this alignment, but only 70 of these ( $10 \%$ of the ICRF) possess the high astrometric quality required to ensure the highest accuracy in the alignment. Accordingly, the current number of VLBI sources for accurately aligning the ICRF and the future Gaia frame is not sufficient. We also showed that the QSOs that will have the most accurate positions measured with Gaia are not those that have the best astrometric positions in the ICRF statistically.

To compensate for the lack of high astrometric quality extragalactic radio sources for this alignment, the VCS catalog, along with the future ICRF-2, will be of high interest. An additional direction would be to identify new appropriate VLBI sources from current deep radio surveys. These solutions mostly concern the northern hemisphere, since the VLBI arrays and observations are concentrated in this part of the world. However, to ensure a homogeneous sky coverage, a major effort is also necessary in the southern hemisphere, most specifically for the declinations below $-40^{\circ}$. To this end, the Asia Pacific Telescope (Gulyaev \& Natusch 2007) is likely to play a significant role.

Acknowledgements. The first author is grateful to the CNES (Centre National d'Études Spatiales, France) for the post-doctoral position granted at Bordeaux Observatory. 


\section{References}

Arias, E. F., Charlot, P., Feissel, M., \& Lestrade J.-F. 1995, A\&A, 303, 604 Beasley, A. J., Gordon, D., Peck, A. B., et al. 2002, ApJS, 141, 13 Bourda, G., Charlot, P., Porcas, R., \& Garrington, S. 2008, in International VLBI Service for Geodesy and Astrometry 2008 General Meeting Proceedings, ed. D. Behrend, \& K. D. Baver

Charlot, P. 1990, AJ, 99, 1309

Charlot, P. 2004, in International VLBI Service for Geodesy and Astrometry 2004 General Meeting Proceedings, ed. N. R. Vandenberg \& K. D. Baver, NASA/CP-2004-212255, 12

Charlot, P., \& Le Campion, J.-F. 2004, in Scientific Highlights 2004, Journées 2004 SF2A Proceedings, ed. F. Combes, D. Barret, T. Contini, F. Meynadier \& L. Pagani (EdP-Sciences), Conference Series, 425

Charlot, P., Fey, A. L., Ojha, R., \& Boboltz, D. A. 2006, in International VLBI Service for Geodesy and Astrometry 2006 General Meeting Proceedings, ed. D. Behrend, \& K. D. Baver, NASA/CP-2006-214140, 321

Condon, J. J., Cotton, W. D., Greisen, E. W., et al. 1998, AJ, 115, 1693

Fey, A. L., \& Charlot, P. 1997, ApJS, 111, 95

Fey, A. L., \& Charlot, P. 2000, ApJS, 128, 17

Fey, A. L., Ma, C., Arias, E. F., et al. 2004, AJ, 127, 3587

Fomalont, E. B., Petrov, L., MacMillan, D. S., Gordon, D., \& Ma, C. 2003, AJ, 125,2562
Gulyaev, S., \& Natusch, T. 2007, in IAU XXVI, Special Session 5: Astronomy for the Developing World, ed. J. Hearnshaw, \& P. Martinez 53

Kovalev, Y. Y., Petrov, L., Fomalont, E., \& Gordon, D. 2007, AJ, 133, 1236

Kovalev, Y. Y., Lobanov, A. P., Pushkarev, A. B., \& Zensus, J. A. 2008, A\&A, 483, 759

Ma, C., Arias, E. F., Eubanks, T. M., et al. 1998, AJ, 116, 516

Lindegren, L. 2008, in A Giant Step: from Milli- to Micro-arcsecond Astrometry, ed. W. J. Wenjin, I. Platais, \& M. A. C. Perryman (Cambridge University Press), IAU Symp. Proc., 248, 217

Mignard, F. 2002, in Gaia: A European Space Project, ed. O. Bienaymé, \& C. Turon, EAS Publ. Ser, 2, 327

Mignard, F. 2003, in IAU XXV, Joint Discussion 16: The International Celestial Reference System: Maintenance and Future Realization, ed. R. Gaume, D. McCarthy, \& J. Souchay, 133

Ojha, R., Fey, A. L., Johnston, K. J., et al. 2004, AJ, 127, 3609

Ojha, R., Fey, A. L., Charlot, P., et al. 2005, AJ, 130, 2529

Perryman, M. A. C., de Boer, K. S., Gilmore, G., et al. 2001, A\&A, 369, 339

Petrov, L., Kovalev, Y., Fomalont, E., \& Gordon, D. 2005, AJ, 129, 1163

Petrov, L., Kovalev, Y., Fomalont, E., \& Gordon, D. 2006, AJ, 131, 1872

Petrov, L., Kovalev, Y., Fomalont, E., \& Gordon, D. 2008, AJ, 136, 580

Schluter, W., \& Behrend, D. 2007, J. Geod., 81, 379

Souchay, J., Gontier, A.-M., \& Barache, C. 2006, A\&A, 453, 743

Véron-Cetty, M.-P., \& Véron, P. 2003, A\&A, 412, 399

Véron-Cetty, M.-P., \& Véron, P. 2006, A\&A, 455, 773 
G. Bourda et al.: Astrometric suitability of ICRF sources for the alignment with Gaia, Online Material $p 1$

Table 3. List of the 70 ICRF extragalactic radio sources suitable for the alignment with the future Gaia frame. The column labelled SI gives the $X$-band structure index of the source. The column labelled Cat. details if the source is categorized in the ICRF as defining (d), candidate (c), other (o) or new (n); and the columns labelled VV2006 name, Type and $V$ indicate respectively (i) the name of the source in the optical catalog Véron \& Véron (2006), (ii) the source type (quasar (Q), BL Lac object (B) or active galaxy (A)), and (iii) its apparent optical magnitude.

\begin{tabular}{|c|c|c|c|c|c|c|c|c|c|c|c|c|}
\hline \multirow[t]{2}{*}{ ICRF designation } & \multirow[t]{2}{*}{ IERS name } & \multirow[t]{2}{*}{$\overline{\overline{\text { SI }}}$} & \multirow[t]{2}{*}{ 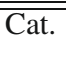 } & \multicolumn{3}{|c|}{$\alpha$} & \multicolumn{3}{|c|}{$\overline{\bar{\delta}}$} & \multirow[t]{2}{*}{ VV2006 name } & \multirow[t]{2}{*}{$\overline{\text { Type }}$} & \multirow[t]{2}{*}{$\bar{V}$} \\
\hline & & & & $\mathrm{h}$ & $\min$ & $\mathrm{sec}$ & $\circ$ & & $"$ & & & \\
\hline $\mathrm{J} 001031.0+105829$ & $0007+106$ & 1 & $\mathrm{~d}$ & 0 & 10 & 31.0059 & 10 & 58 & 29.504 & III Zw 2 & $\bar{A}$ & 15.40 \\
\hline $\mathrm{J} 001331.1+405137$ & $0010+405$ & 2 & $\mathrm{~d}$ & 0 & 13 & 31.1302 & 40 & 51 & 37.144 & 4C 40.01 & Q & 17.90 \\
\hline J005041.3-092905 & 0048-097 & 1 & o & 0 & 50 & 41.3174 & -9 & 29 & 5.210 & PKS 0048-09 & B & 17.44 \\
\hline $\mathrm{J} 011205.8+224438$ & $0109+224$ & 2 & $\mathrm{~d}$ & 1 & 12 & 5.8247 & 22 & 44 & 38.786 & S2 $0109+22$ & B & 15.66 \\
\hline J020504.9+321230 & $0202+319$ & 2 & d & 2 & 5 & 4.9254 & 32 & 12 & 30.096 & DW $0202+31$ & $\mathrm{Q}$ & 17.40 \\
\hline J021046.2-510101 & $0208-512$ & 2 & o & 2 & 10 & 46.2004 & -51 & 1 & 1.892 & PKS 0208-512 & B & 16.93 \\
\hline J021748.9+014449 & $0215+015$ & 1 & d & 2 & 17 & 48.9547 & 1 & 44 & 49.699 & PKS 0215+015 & Q & 16.09 \\
\hline J023838.9+163659 & $0235+164$ & 1 & $\mathrm{~d}$ & 2 & 38 & 38.9301 & 16 & 36 & 59.275 & AO $0235+164$ & Q & 15.50 \\
\hline J030335.2+471616 & $0300+470$ & 2 & $\mathrm{o}$ & 3 & 3 & 35.2422 & 47 & 16 & 16.276 & 4C 47.08 & B & 16.95 \\
\hline J031301.9+412001 & $0309+411$ & 2 & $\mathrm{~d}$ & 3 & 13 & 1.9621 & 41 & 20 & 1.184 & NRAO 128 & A & 18.00 \\
\hline J033413.6-400825 & $0332-403$ & 1 & o & 3 & 34 & 13.6545 & -40 & 8 & 25.398 & PKS 0332-403 & B & 17.50 \\
\hline J040534.0-130813 & $0403-132$ & 1 & o & 4 & 5 & 34.0034 & -13 & 8 & 13.691 & PKS 0403-13 & $\mathrm{Q}$ & 17.09 \\
\hline J040748.4-121136 & $0405-123$ & 2 & $\mathrm{c}$ & 4 & 7 & 48.4310 & -12 & 11 & 36.660 & PKS 0405-12 & $\mathrm{Q}$ & 14.86 \\
\hline $\mathrm{J} 042446.8+003606$ & $0422+004$ & 2 & $\mathrm{~d}$ & 4 & 24 & 46.8421 & 0 & 36 & 6.330 & PKS 0422+00 & B & 16.98 \\
\hline $\mathrm{J} 050842.3+843204$ & $0454+844$ & 2 & $\mathrm{~d}$ & 5 & 8 & 42.3635 & 84 & 32 & 4.544 & S5 $0454+84$ & B & 16.50 \\
\hline J045550.7-461558 & $0454-463$ & 1 & $\mathrm{c}$ & 4 & 55 & 50.7725 & -46 & 15 & 58.682 & PKS 0454-46 & Q & 16.90 \\
\hline J050643.9-610940 & $0506-612$ & 2 & $\mathrm{~d}$ & 5 & 6 & 43.9887 & -61 & 9 & 40.993 & PKS 0506-61 & Q & 16.85 \\
\hline J053007.9-250329 & $0528-250$ & 2 & o & 5 & 30 & 7.9628 & -25 & 3 & 29.900 & PKS 0528-250 & Q & 17.34 \\
\hline J060940.9-154240 & $0607-157$ & 2 & $\mathrm{c}$ & 6 & 9 & 40.9495 & -15 & 42 & 40.673 & PKS 0607-15 & $\mathrm{Q}$ & 18.00 \\
\hline J064204.2+675835 & $0636+680$ & 1 & $\mathrm{~d}$ & 6 & 42 & 4.2574 & 67 & 58 & 35.621 & S4 0636+68 & $\mathrm{Q}$ & 16.60 \\
\hline J072153.4+712036 & $0716+714$ & 1 & d & 7 & 21 & 53.4485 & 71 & 20 & 36.363 & S5 0716+71 & B & 15.50 \\
\hline J075706.6+095634 & $0754+100$ & 2 & $\mathrm{~d}$ & 7 & 57 & 6.6429 & 9 & 56 & 34.852 & PKS 0754+100 & B & 15.00 \\
\hline J081126.7+014652 & $0808+019$ & 1 & $\mathrm{c}$ & 8 & 11 & 26.7073 & 1 & 46 & 52.220 & PKS 0808+019 & B & 17.20 \\
\hline J082601.5-223027 & $0823-223$ & 1 & $\mathrm{c}$ & 8 & 26 & 1.5729 & -22 & 30 & 27.204 & PKS 0823-223 & B & 16.20 \\
\hline J083052.0+241059 & $0827+243$ & 2 & $\mathrm{c}$ & 8 & 30 & 52.0862 & 24 & 10 & 59.820 & B2 0827+24 & Q & 17.26 \\
\hline J083740.2+245423 & $0834+250$ & 2 & $\mathrm{n}$ & 8 & 37 & 40.2457 & 24 & 54 & 23.122 & B2 $0834+25$ & Q & 17.90 \\
\hline J091552.4+293324 & $0912+297$ & 1 & $\mathrm{~d}$ & 9 & 15 & 52.4016 & 29 & 33 & 24.043 & B2 $0912+29$ & B & 16.39 \\
\hline J095533.1+690355 & $0951+693$ & 1 & o & 9 & 55 & 33.1731 & 69 & 3 & 55.061 & NGC 3031 & Q & 11.63 \\
\hline J095847.2+653354 & $0954+658$ & 2 & $\mathrm{~d}$ & 9 & 58 & 47.2451 & 65 & 33 & 54.818 & S4 0954+65 & B & 16.81 \\
\hline J095820.9+322402 & $0955+326$ & 2 & $\mathrm{~d}$ & 9 & 58 & 20.9496 & 32 & 24 & 2.209 & 3 C 232 & Q & 15.78 \\
\hline $\mathrm{J} 101447.0+230116$ & $1012+232$ & 2 & $\mathrm{~d}$ & 10 & 14 & 47.0654 & 23 & 1 & 16.571 & PKS 1011+23 & Q & 17.80 \\
\hline $\mathrm{J} 102444.8+191220$ & $1022+194$ & 2 & $\mathrm{c}$ & 10 & 24 & 44.8096 & 19 & 12 & 20.416 & 4C 19.34 & $\mathrm{Q}$ & 17.49 \\
\hline $\mathrm{J} 104423.0+805439$ & $1039+811$ & 2 & $\mathrm{~d}$ & 10 & 44 & 23.0626 & 80 & 54 & 39.443 & S5 1039+81 & Q & 17.90 \\
\hline $\mathrm{J} 110427.3+381231$ & $1101+384$ & 2 & $\mathrm{c}$ & 11 & 4 & 27.3139 & 38 & 12 & 31.799 & MARK 421 & B & 12.90 \\
\hline $\mathrm{J} 110331.5-325116$ & $1101-325$ & 2 & $\mathrm{c}$ & 11 & 3 & 31.5264 & -32 & 51 & 16.692 & PKS 1101-325 & Q & 16.30 \\
\hline J111358.6+144226 & $1111+149$ & 2 & $\mathrm{~d}$ & 11 & 13 & 58.6951 & 14 & 42 & 26.953 & PKS 1111+149 & Q & 17.90 \\
\hline $\mathrm{J} 112553.7+261019$ & $1123+264$ & 2 & $\mathrm{o}$ & 11 & 25 & 53.7119 & 26 & 10 & 19.979 & PKS 1123+26 & Q & 18.00 \\
\hline J114658.2+395834 & $1144+402$ & 1 & $\mathrm{o}$ & 11 & 46 & 58.2979 & 39 & 58 & 34.305 & S4 1144+40 & Q & 18.00 \\
\hline $\mathrm{J} 115019.2+241753$ & $1147+245$ & 2 & $\mathrm{~d}$ & 11 & 50 & 19.2122 & 24 & 17 & 53.835 & B2 $1147+24$ & $\mathrm{~B}$ & 15.74 \\
\hline $\mathrm{J} 121752.0+300700$ & $1215+303$ & 2 & $\mathrm{~d}$ & 12 & 17 & 52.0820 & 30 & 7 & 0.636 & B2 $1215+30$ & $\mathrm{~B}$ & 15.62 \\
\hline $\mathrm{J} 122222.5+041315$ & $1219+044$ & 2 & $\mathrm{~d}$ & 12 & 22 & 22.5496 & 4 & 13 & 15.776 & PKS 1219+04 & Q & 17.98 \\
\hline $\mathrm{J} 122503.7+125313$ & $1222+131$ & 2 & $\mathrm{n}$ & 12 & 25 & 3.7433 & 12 & 53 & 13.139 & NGC 4374 & A & 12.31 \\
\hline $\mathrm{J} 125438.2+114105$ & $1252+119$ & 2 & $\mathrm{~d}$ & 12 & 54 & 38.2556 & 11 & 41 & 5.895 & PKS 1252+11 & Q & 16.64 \\
\hline $\mathrm{J} 133245.2+472222$ & $1330+476$ & 1 & $\mathrm{n}$ & 13 & 32 & 45.2464 & 47 & 22 & 22.668 & B3 $1330+476$ & Q & 17.96 \\
\hline J135256.5-441240 & $1349-439$ & 2 & $\mathrm{c}$ & 13 & 52 & 56.5349 & -44 & 12 & 40.387 & PKS 1349-439 & B & 16.37 \\
\hline $\mathrm{J} 141908.1+062834$ & $1416+067$ & 2 & d & 14 & 19 & 8.1802 & 6 & 28 & 34.803 & 3C 298.0 & Q & 16.79 \\
\hline $\mathrm{J} 142230.3+322310$ & $1420+326$ & 1 & c & 14 & 22 & 30.3790 & 32 & 23 & 10.440 & B2 $1420+32$ & Q & 17.50 \\
\hline $\mathrm{J} 142700.3+234800$ & $1424+240$ & 2 & $\mathrm{c}$ & 14 & 27 & 0.3918 & 23 & 48 & 0.038 & PKS 1424+240 & B & 14.95 \\
\hline $\mathrm{J} 151053.5-054307$ & $1508-055$ & 2 & $\mathrm{n}$ & 15 & 10 & 53.5914 & -5 & 43 & 7.417 & PKS 1508-05 & Q & 17.21 \\
\hline J152237.6-273010 & $1519-273$ & 1 & $\mathrm{c}$ & 15 & 22 & 37.6760 & -27 & 30 & 10.785 & PKS 1519-273 & B & 17.70 \\
\hline $\mathrm{J} 154049.4+144745$ & $1538+149$ & 2 & $\mathrm{~d}$ & 15 & 40 & 49.4915 & 14 & 47 & 45.885 & 4C 14.60 & Q & 17.30 \\
\hline J154929.4+023701 & $1546+027$ & 2 & c & 15 & 49 & 29.4368 & 2 & 37 & 1.164 & PKS 1546+027 & Q & 17.45 \\
\hline J163813.4+572023 & $1637+574$ & 2 & $\mathrm{~d}$ & 16 & 38 & 13.4563 & 57 & 20 & 23.979 & OS 562 & Q & 16.90 \\
\hline $\mathrm{J} 172824.9+042704$ & $1725+044$ & 2 & $\mathrm{~d}$ & 17 & 28 & 24.9527 & 4 & 27 & 4.914 & PKS 1725+044 & $\mathrm{Q}$ & 16.99 \\
\hline $\mathrm{J} 172818.6+501310$ & $1727+502$ & 2 & $\mathrm{~d}$ & 17 & 28 & 18.6239 & 50 & 13 & 10.470 & I Zw 187 & B & 15.97 \\
\hline $\mathrm{J} 175132.8+093900$ & $1749+096$ & 1 & $\mathrm{c}$ & 17 & 51 & 32.8186 & 9 & 39 & 0.729 & OT 081 & Q & 16.78 \\
\hline J180132.3+440421 & $1800+440$ & 1 & $\mathrm{~d}$ & 18 & 1 & 32.3149 & 44 & 4 & 21.900 & B3 $1800+440$ & Q & 17.90 \\
\hline J184916.0+670541 & $1849+670$ & 1 & $\mathrm{~d}$ & 18 & 49 & 16.0723 & 67 & 5 & 41.680 & S4 1849+67 & Q & 16.90 \\
\hline
\end{tabular}


G. Bourda et al.: Astrometric suitability of ICRF sources for the alignment with Gaia, Online Material p 2

Table 3. continued.

\begin{tabular}{|c|c|c|c|c|c|c|c|c|c|c|c|c|}
\hline \multirow[t]{2}{*}{ ICRF designation } & \multirow[t]{2}{*}{ IERS name } & \multirow[t]{2}{*}{$\overline{\overline{\text { SI }}}$} & \multirow[t]{2}{*}{ Cat. } & \multicolumn{3}{|c|}{$\bar{\alpha}$} & \multicolumn{3}{|c|}{$\overline{\bar{\delta}}$} & \multirow[t]{2}{*}{ "VV2006 name } & \multirow[t]{2}{*}{ Type } & \multirow[t]{2}{*}{$\bar{V}$} \\
\hline & & & & $\mathrm{h}$ & $\min$ & $\mathrm{sec}$ & $\circ$ & & " & & & \\
\hline $\mathrm{J} 192332.1-210433$ & $1920-211$ & 2 & $\mathrm{c}$ & 19 & 23 & 32.1898 & -21 & 4 & 33.333 & TEX 1920-211 & $\mathrm{Q}$ & 17.50 \\
\hline J195759.8-384506 & $1954-388$ & 2 & $\mathrm{~d}$ & 19 & 57 & 59.8193 & -38 & 45 & 6.356 & PKS 1954-388 & Q & 17.07 \\
\hline $\mathrm{J} 210138.8+034131$ & $2059+034$ & 2 & $\mathrm{~d}$ & 21 & 1 & 38.8342 & 3 & 41 & 31.322 & PKS 2059+034 & Q & 17.78 \\
\hline $\mathrm{J} 212912.1-153841$ & $2126-158$ & 2 & $\mathrm{c}$ & 21 & 29 & 12.1759 & -15 & 38 & 41.041 & PKS 2126-15 & Q & 17.00 \\
\hline $\mathrm{J} 214622.9-152543$ & $2143-156$ & 2 & $\mathrm{~d}$ & 21 & 46 & 22.9793 & -15 & 25 & 43.885 & PKS 2143-156 & $\mathrm{Q}$ & 17.27 \\
\hline $\mathrm{J} 215852.0-301332$ & $2155-304$ & 2 & $\mathrm{c}$ & 21 & 58 & 52.0651 & -30 & 13 & 32.118 & PKS 2155-304 & B & 13.09 \\
\hline J222940.0-083254 & $2227-088$ & 1 & $\mathrm{c}$ & 22 & 29 & 40.0843 & -8 & 32 & 54.435 & PKS 2227-08 & Q & 17.43 \\
\hline $\mathrm{J} 225307.3+194234$ & $2250+190$ & 2 & $\mathrm{n}$ & 22 & 53 & 7.3692 & 19 & 42 & 34.629 & HS $2250+1926$ & Q & 18.00 \\
\hline $\mathrm{J} 225717.5+024317$ & $2254+024$ & 1 & $\mathrm{c}$ & 22 & 57 & 17.5631 & 2 & 43 & 17.512 & PKS $2254+024$ & $\mathrm{Q}$ & 17.96 \\
\hline $\mathrm{J} 225717.3+074312$ & $2254+074$ & 1 & $\mathrm{~d}$ & 22 & 57 & 17.3031 & 7 & 43 & 12.303 & PKS 2254+074 & B & 16.36 \\
\hline $\mathrm{J} 225805.9-275821$ & $2255-282$ & 2 & $\mathrm{o}$ & 22 & 58 & 5.9629 & -27 & 58 & 21.257 & PKS 2255-282 & $\mathrm{Q}$ & 16.77 \\
\hline $\mathrm{J} 230343.5-680737$ & $2300-683$ & 2 & $\mathrm{c}$ & 23 & 3 & 43.5663 & -68 & 7 & 37.462 & PKS 2300-683 & $\mathrm{Q}$ & 16.38 \\
\hline
\end{tabular}

